DOI - https://doi.org/10.5965/2316796309172020058

\title{
UX Heuristic evaluation of cross-channel interaction systems: first experimentationsd
}

Adriano Bernardo Renzi ${ }^{1}$

Olney Almeida ${ }^{2}$ 


\section{Abstract}

This is the first experimentation using the 9 UX heuristics (Renzi 2016) as a guide for a cross-channel system ecology heuristic evaluation. The paper presents the preparations, proceedings and results of the Airbnb system ecology UX evaluation, permeating three digital devices - smartphone, desktop computer and tablet - in order to simulate a cross-channel user experience journey in four micro-moments.

Key-words: Interaction, User Experience, UX heuristics, Heuristic evaluation

\section{Third wave of computing}

As the 3rd wave of computing advances and evolves, the fulfillment of experiences is increasingly related to journeys permeating different channels (Renzi 2016). Users go through diverse apparatuses to help complete their objectives and digital interactions are gradually integrated into daily common artifacts - forming an Internet of Things.

The idea of cross-channel interaction starts, based on the evolution from traditional information architecture concepts to the pervasive information architecture manifest of Resmini and Rosatti (2011), as ubiquitous computing advances. The authors explicit the necessity of perspective change, in which the information architecture should not be restricted to the web, but must advance to spaces, ambiances, services and shared process projects, evolving from human-computer interaction to humaninformation interaction.

The information architecture should connect different contexts and medias in one cross-channel system ecology, where artifacts are not an isolated interaction, but have interoperability between them to create a cross-channel experience (Renzi 2016).

According to Resmini and Lacerda (2016), the cross-channel ecosystem is the "actor-created transient environments straddling non-contiguous digital and physical spaces, devices, services, and people that constitute the environment in which people form intentions, take actions, and where their experiences unfold".

For instance, it is necessary to go beyond interactions with isolated digital artifacts and think the whole ecology system (Renzi 2016, 2017) to better understand how users' journey experiences (fig.1) are built in specific contexts. The user experience becomes a narrative journey, involving actions in the physical world while using many different digital devices linked together by a dynamic ecology, with connections that brings together one whole story (Renzi 2017). Each part of the narrative - a short story - can encompass different digital artifacts accessing the same ecology system, influenced by context of use and physical environment.

Figure 1: pervasive experience through different devices to build an experience journey (Renzi 2016)

\section{ISSN: 2316-7963}

\footnotetext{
${ }^{1}$ Doutor em Design, ESDI/UERJ (adrianorenzi@gmail.com)
}

2 SENAC (nei.fda@gmail.com)
The connection between physical artifacts that do not share the same location allows systems "to access remote sensor data and to control the physical world from a distance", giving rise to "new synergistic services that go beyond the services that can be provided by an isolated embedded system" (Kopetz 2011).

From the philosophy of information perspective, Floridi (2010) defines as infosphere, a complex "environment constituted by all informational entities (and) 
thus including informational agents as well, their properties, interactions, processes and mutual relations". The author (ibidem) identifies in design the primary way to counterbalance technological pushes in the infosphere: "design is not limited to the simple production of value, but plays a larger role in building an information ethics for the whole infosphere and all its components and inhabitants", including "artificial, digital, or synthetic" (Floridi 2014).

The UX designer has to understand the whole journey in order to plan possible touchpoints of interactions and create service or product experiences, integrated by different channels: a pervasive cross-channel experience.

As usability evaluations related to interface interactions (originated in the 2 nd wave of computing) focus mostly on isolated systems, the evolution of the 3rd wave of computing into pervasive cross-channel interactions has urged new concepts and evaluation proposals. The technology advancements brought new usability evaluations for new specific devices, with new types of interaction and new systems. Systems for smartphones, tabletops, tablets and gestural sensor environments could properly have their usability measured. However adapted to new technology possibilities, were still evaluations of interactions with one isolated system, without thinking the whole narrative experience.

\section{Evaluating cross-channel scenarios}

The development of new devices and the evolution of interaction brings new proposals based on Nielsen and Molich's heuristics evaluation from the 90's (second wave of computing): Apted et al. (2009) propose heuristics focused on tabletop interactions, Inostroza et al. $(2013,2016)$ present heuristics for smartphones and Neto interactions, Inostroza et al. $(2013,2016)$ present heuristics for smartphones and Neto heuristics proposal are adapted to guide and evaluate isolated devices, based on type of interaction, context and size of display.

In order to analyze the whole experience journey and the cross-channel interaction within one integrated ecology system, a set of 9 UX heuristics was proposed in 2016 (Renzi), encompassing interface cognitive perception, 5-sense interactions, pervasive information and interoperability between systems. The basic description of each of the 9 UX heuristics follows:

- Place-making: refers to the self-localization of users in the system and within the experience journey. Visual interaction, hierarchical layout and structure, as well as physical environment should facilitate the user's understanding of where he is. Since users most likely will use different devices and the ambience itself to fulfill objectives, it is important that physical environments also be part of the user experience strategies to create an integrated journey.

2 - Consistency: the system has to present visual, typographic, information, actions and interaction consistency. If a user utilizes different devices to execute partial actions of the whole experience, each touchpoint access has to present the same rules and responses to actions, independent of the artifact that is being used within the journey.

3 - Resilience: flexibility of the interaction flow and touchpoints in order to adequate to different users, different journey strategies and different contexts of use. The interactive ambiance and the system structure should be prepared to search, interaction and journey diverse strategies by different users, sometimes with distinct roles in the same journey.

4 - Reduction - even if the back-end of the system is complex in its structure, the options and the contents have to be presented to users in objective way and with simple usage, providing reduced interactive actions and minimum cognitive workload in their journey. From the users' point of view, the path of possible actions has to be obvious to their necessities.

5 - Correlation: the system has to go beyond the semantic correlation exposed by Lévy and Resmini and Rosatti, to help users find information and content naturally The principle expands to a correlation of data between distinct points of interaction and apparatuses, as well as correlation of actions from different users within the same experience journey. One action started in one device should be easily continued in any other device linked to the system ecology.

6 - Equivalency to cultural conventions: it is important to understand users' references regarding technology, processes, functionality comprehension and interactions, to use as a base in the development of a new system. To create structures and interactions that users are not familiar with can surface doubts and misunderstanding about the system.

7 - Visual intuitive content: users must recognize functionalities, hierarchy, pathways and information with minimal memory load, by making objects, actions and options easy to recognize and understand;

8 - Natural, intuitive and direct interactions: any touchpoint of interaction with the system should be as intuitive as possible, by direct gestural manipulation, objective simple vocal commands or though.

9 - Contextual ergonomics: physical environments, ergonomic reach in each device involved, contexts of use within the journey of experience and human physical limitations should be considered while projecting touchpoints of interaction with the system.

This paper presents the first experiment using the $9 \mathrm{UX}$ heuristics as basis for a cross-channel system heuristic evaluation. This specific experiment is part of a broader research project that compares different evaluation processes in identifying problems in the Airbnb system throughout all its channels of interaction.

The company has over 3 million active places for renting in more than 191 countries and 65 thousand cities. Over the years, more than 100 million guests have used at least one of Airbnb's affiliated hosts. Since 2017, the company started to be more than just connecting people to places to stay while traveling, and increased its portfolio to connect people to experiences: places, physical activities (fig. 2), touristic visits, local culture workshops, music and gastronomy. Users can reach the Airbnb ecology system and book an experience, or an apartment, through 3 different channels: computer (website), smartphone (app) and tablet (app). 
Figure 2: Example of Airbnb experience service: surf school in Bali. MySurf School Bal

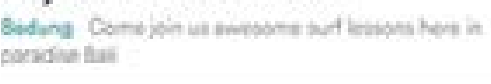

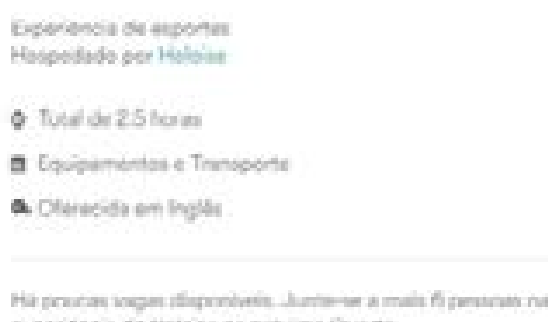

(5)
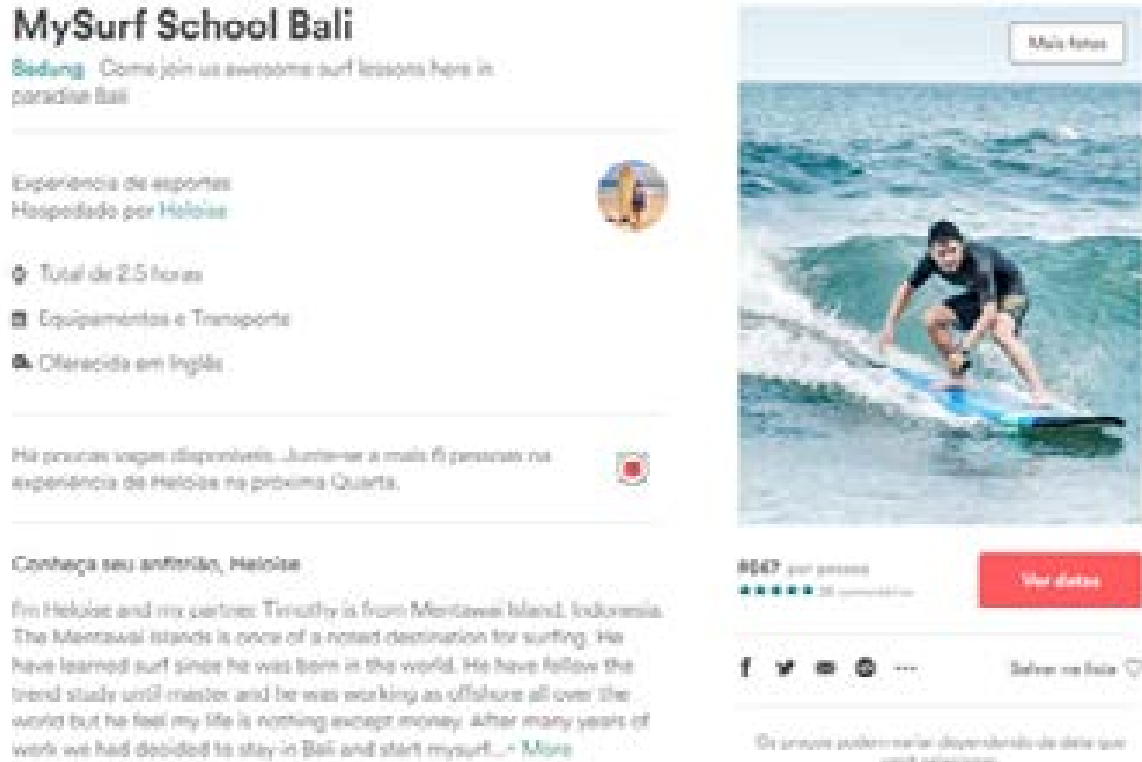

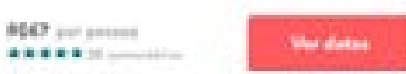

f

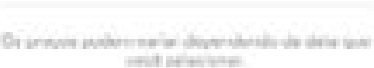

Using the company' system as object of study seems a great opportunity to test the UX heuristics (as well as other heuristic evaluations), as its system ecology intends to offer a pervasive cross-channel interaction and to build an experience journey.

\section{UX heuristic evaluation}

Following Nielsen's heuristic evaluation proposal from the 90's, the heuristic evaluation consists of experts going through a system to analyze and evaluate its interactions based on a set of heuristics.

For this experiment, the invitee had to be UX experts (UX, not usability) with previous experiences in evaluating systems using the traditional heuristic evaluation, as well as understanding the pervasive cross-channel concept. Experts with experience in other usability methods, such as think-aloud protocol, did not count if they have never applied a heuristic evaluation before.

According to Nielsen's experiment in 1992, with 19 usability experts, the use of heuristic evaluation showed that 3-5 experts could bring up around $75 \%$ of the tota usability errors (fig. 3). For this first attempt, 3 UX experts with diverse background agreed to participate:

1. Doctor of Sciences, specialized in UX strategy, technology and Human-computer interaction.

2. UX designer, with specialization in interaction design, Al and chat bots.

3. Master of Arts, specialized in Education and Technology

The method was organized in 3 sequential steps, after participants accepted the invitation and visited the system for an overall view:

Figure 3: Nielsen's relation between number of experts and percentage of errors found (Nielsen 1995).

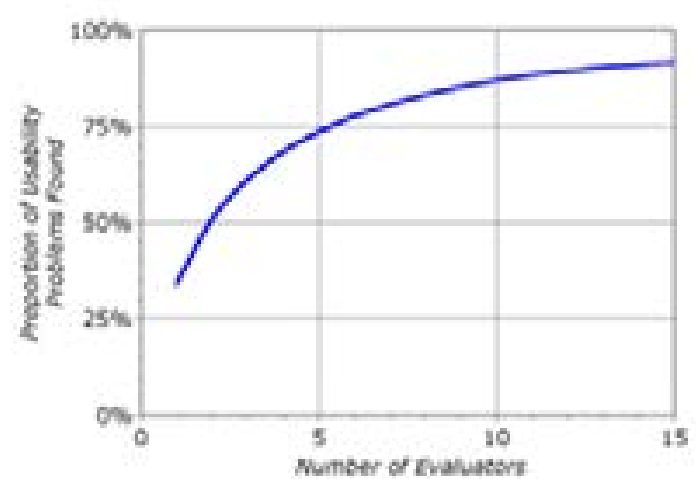

- Step 1: experts receive an evaluating sheet with directions about proceedings and the 9 heuristics descriptions. Since these heuristics are very different from the well-known usability heuristics, it is advisable to contact directly each expert to explain the concept and go through all heuristics to avoid misunderstandings and distorted results.

- Step 2: experts analyze the system ecology, following the given directions, and mark all problems that are found during the analysis on the evaluating sheet. Each $\mathrm{UX}$ problem is described and related to its correspondent heuristics. This evaluation is usually done and marked by the expert alone. After all evaluation is marked, the expert sends the documentation back to the researcher.

- Step 3: the researcher collects all documents from all the experts and compiles all problems into one single document. This single document, with all noted problems, is sent separately back to each expert in order to have them indicate the gravity of each problem.

If the evaluation execution is face-to-face (Renzi and Freitas 2013), each expert must indicate the gravity of each problem found in the evaluating sheet, to better compile all information. As seen in previous research with heuristic evaluation (ibidem 2013), a face-to-face evaluation can be applied if the participating expert showns schedule problems. The advantage of a face-to-face evaluation is the availability of the researcher to clarify any doubts about the process.

After all problems are rated by their gravity, the researcher calculates the average value and set the final results. The experts can be brought up together to discuss problems and gravities, if reaching an agreement is needed.

For this experiment, the gravity scale was based on Nielsen's original heuristic evaluation proposal. According to Nielsen's heuristic evaluation (followed by Apted et al., Inostrozza et al., Neto and Campos), the gravity of problems varies from 0 (zero) to 4 (four):

- Gravity 0 (zero): It is not a problem. Usually pointed to parts of the system or interface that could be considered awkward, but does not get in the way of users.

- Gravity 1 (one): It is an aesthetic problem. Pointed mostly to parts of the system related interface layout that may not follow users expectations or are considered an aesthetic flaw, but do not affect users interactions or interoperability of information

- Gravity 2 (two): It is a minor problem. These are problems that cause discomfort to users, may bring misunderstandings of information, result in interaction or action confusion, oblige users to re-do or take longer actions, or could distort the notion 
of place-making. These problems do not impede procedures nor may cause serious interaction problems, but certainly are problems that bring discomfort and irritability to users.

- Gravity 3 (three): It is a major problem. These are problems that may diverge users from their objectives. They can take forms such as misleading information or interactions, actions requirements that are not obvious or are completely different from other channels, lack of interoperability between channels, information or action break, functionalities that are far from users expectations or do not follow a set pattern or have low affordance. These problems can make users give up using the system or try any other option out of it.

- Gravity 4 (four): It is a catastrophic problem. These are problems that may impede users to reach objectives, or compromise the whole system structure, or interfere seriously in users' sense of place-making. Mostly, these are problems instated in the system's concept, structure, taxonomy, interoperability and information system that affects the whole idea of cross-channel interaction, or sometimes just isolated interactions. These are serious problems that can bring companies to re-think the whole idea of a product.

For this first experiment, the experts used an evaluating sheet to include the related gravities at first analysis and the researcher calculated and categorized the final results. Facing any doubts, the researcher would contact experts for clarification.

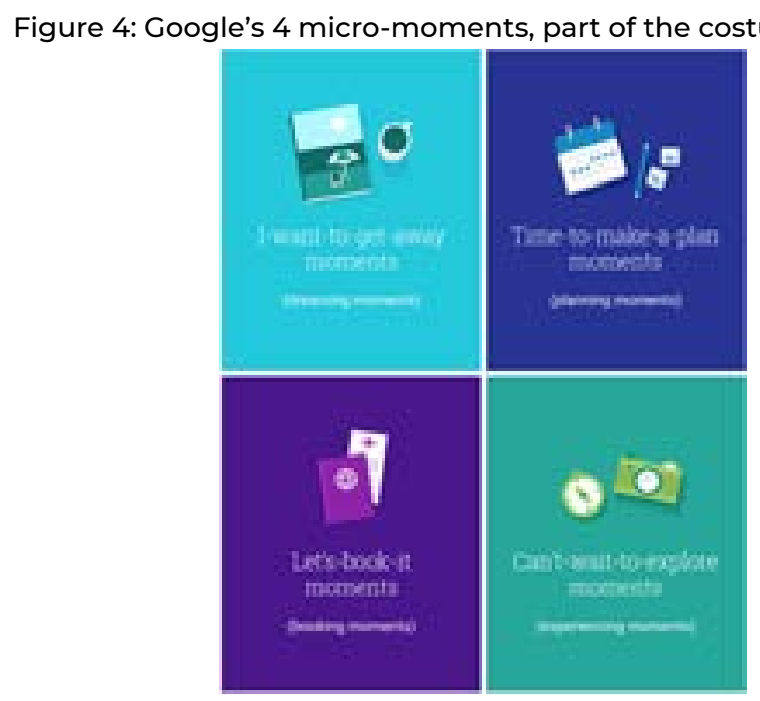

According to Google's article about traveling, the costumer journey follows 4 micro-moments: dreaming moment (I want to get away), planning moment (time to make a plan), booking moment (let's book it) and experience moment (can't wait to explore). Previous research results from interviews (Renzi et al. 2018), confirmed Google's insights on travel costumer journey (fig.4) and helped understand the user's experience journey, as well as, set the order for this experiment. The evaluation tried to encompass the planning, booking and experience moments, following the interview results, to set the digital apparatuses and their order, for interaction and analysis.

Each expert had to follow actions, distributed throughout different devices (smartphone, desktop and tablet), in 4 steps (fig.5):
Figure 5: Planned pervasive actions for evaluation, following users' travel journey (Renzi et al. 2018).

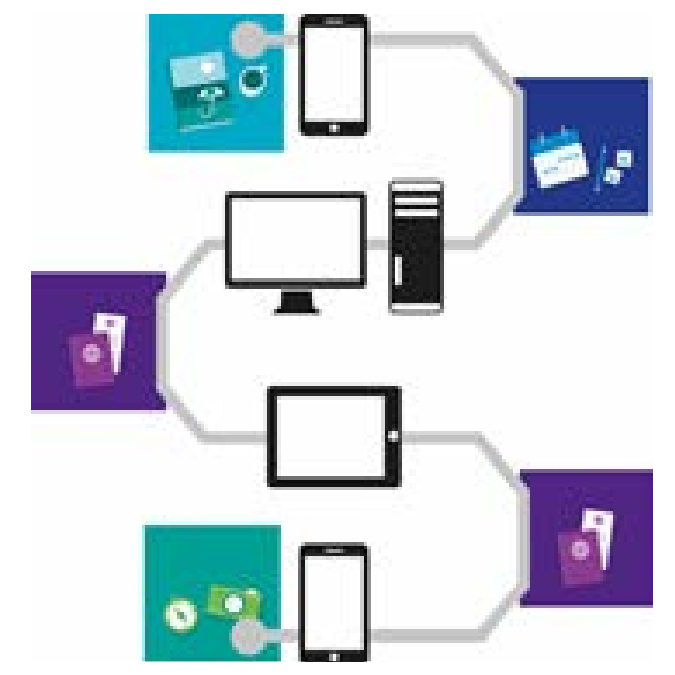

- Step 1: (smartphone) enter the Airbnb app and search for Paraíso Village lodging located in Rio de Janeiro, and its availability between December 28th and January 3rd. Analyze all information regarding the place and save it.

- Step 2: (desktop) login on Airbnb website, access your saved places and locate Paraíso Village lodging. Analyze all displayed information, compare to previous step. Send a message to the owner or manager of lodge.

- Step 3: (tablet) login on Airbnb app for tablets and look for new messages from the owner. Check all information, compare interaction, information and actions to previous steps and book the chosen place.

- Step 4: (smartphone) enter the Airbnb app, access the booking information and verify the location information, simulating the arrival at destiny. Analyze and compare information, actions and interactions to previous steps.

\section{Results}

The UX heuristic evaluation pointed a total of 81 problems, distributed through smartphone, desktop and tablet. Most part of problems found $(23,5 \%)$ was related to heuristic 7 (visual intuitive content: users must recognize functionalities, hierarchy, pathways and information with minimal memory load, by making objects, actions and options easy to recognize and understand).

The heuristic 7 occurs 9 times using the Airbnb app for smartphone, 6 times while using the company's website on desktop and 4 times while using the Airbnb app for the tablet to fulfill the journey. The gravity of problems related to heuristic 7 was rated mostly as minor problems and major problems (fig.6), as it is expected in heuristic evaluations. But 1 catastrophic problem related to the heuristic is noted using desktop. 
Figure 6: Problems related to heuristic 7: gravity $\mathrm{x}$ device used.

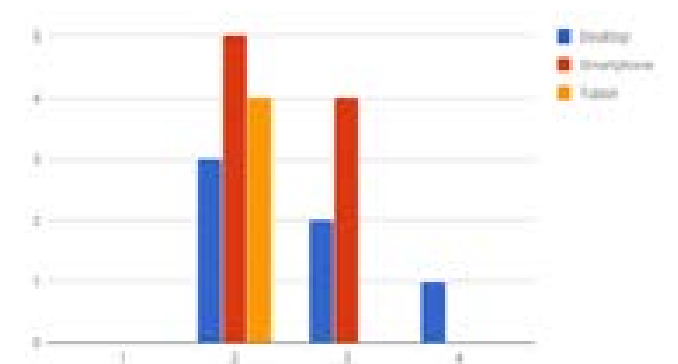

The heuristic 6 (equivalency to cultural conventions: it is important to understand users' references regarding technology, processes, functionality comprehension and interactions, to use as a base in the development of a new system. To create structures and interactions that users are not familiar with can surface doubts and misunderstanding about the system) is the second most related to noted 15 problems (17\%). The heuristic is also more present during the use of smartphones, with 9 noted problems. Desktop use shows 5 problems and tablet just 1 error.

Although not as many errors as heuristic 7, the evaluators considered 5 errors connected to equivalency to cultural conventions to be catastrophic errors (gravity 4): 3 interacting with smartphone and 2 interacting with desktop (fig.7).

Figure 7: Problems related to heuristic 6: gravity $x$ device used.

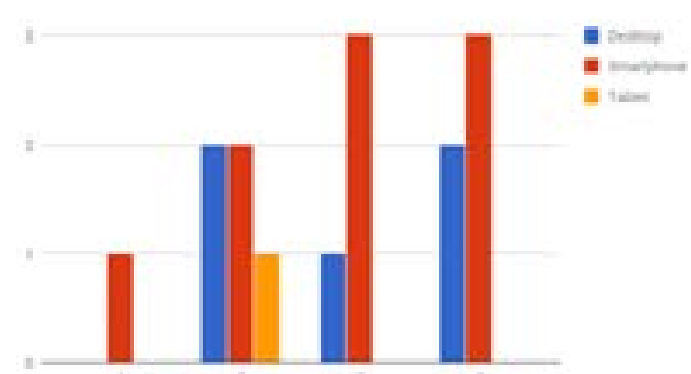

Part of the problems were noted related to information inconsistency during pervasive interaction with different channels, such as, the price of the saved apartment changing from desktop to smartphone. This was considered a catastrophic problem as it may directly infuse doubts about security of the system and make users quit the experience. Evaluators connected this problem to heuristic 6 due to "creating structures and interactions that users are not familiar with can surface doubts and misunderstanding about the system"

Problems related to heuristic 1 (Place-making: refers to the self-localization of users in the system and within the experience journey. Visual interaction, hierarchical layout and structure, as well as physical environment should facilitate the user's understanding of where he is. Since users most likely will use different devices and the ambience itself to fulfill objectives, it is important that physical environments also be part of the user experience strategies to create an integrated journey) numbers third place, totaling 12 problems (fig.8). Following the pattern of previous mentioned heuristics, the Airbnb app for smartphones has the most number of problems, reaching a total of 7 errors.

Figure 8: Problems related to heuristic 1: gravity $x$ device used.

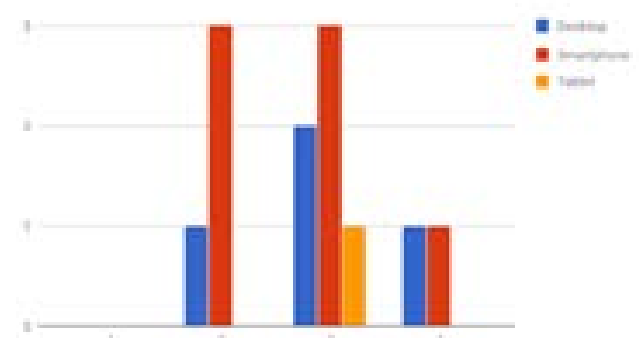

Analyzing the pointed problems by their gravity, catastrophic problems (gravity 4) are pointed related to 5 of 9 heuristics. Most of them are connected to the use of smartphone App (fig.9). The major problems (gravity 3 ) are mostly related to the use of the smartphone app and website through desktop. The tablet App shows presence related to minor problems (gravity 2 ), but still is surpassed by both smartphone and related to minor problems (gravity 2 ), but still is surpassed by both smartphone and
desktop experiences. One of the minor problems noted while using tablet, for example, is the difficulty to find where to login, hidden inside the menu (heuristic 7).

Figure 9: noted catastrophic problems - heuristic incurrence comparison.

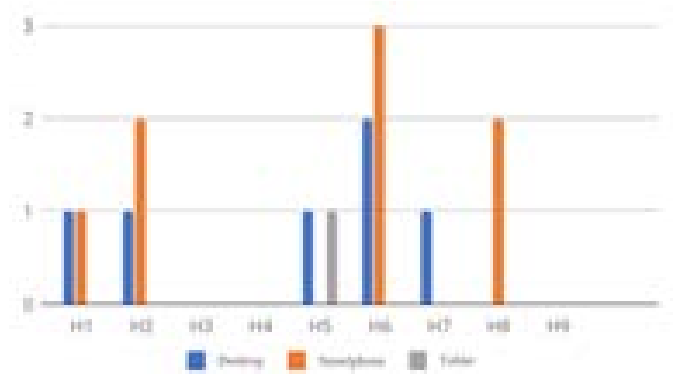

Part of the notified problems became evident during the cross-channel interaction, such as, while using the search tool on desktop it was not possible to find the Paraíso Village lodge, previously found using the smartphone Airbnb App. Not even rewriting the same words. The problem got worse when evaluators proceeded to step 3 (from desktop to tablet) and realized that it was not possible to retrieve the information of Paraíso Village Lodge, which was saved before through smartphone and resaved on desktop. It was necessary to search once again the lodge and save it again. Evaluators considered this error through channels related to heuristics 1 (placemaking) and 3 (resilience: flexibility of the interaction flow and touch points in order to adequate to different users, different journey strategies and different contexts of use. The interactive ambiance and the system structure should be prepared to search, interaction and journey diverse strategies by different users, sometimes with distinct roles in the same journey). 
Another cross-channel problem example regards taxonomy and functionality recognition that affects directly users' sense of self-location within the system: the Airbnb App for tablets do not use the word "saved" to identify places previously saved by the user. Instead, it presents the icon "wish list", which links to a page with saved places, but with no information, nor any possibility of interaction for further details of any specific saved place (fig.10). The evaluators related this problem to heuristic 1 (place-making) due to the loss of self-location, heuristic 4 (reduction - even if the backend of the system is complex in its structure, the options and the contents have to be presented to the users in objective way and with simple usage, providing reduced interactive actions and minimum cognitive workload in their journey. From the users' point of view, the path of possible actions has to be obvious to their necessities), due to the system's unnecessary complexity of interaction to a dead-end page, and heuristic 5 (correlation: the system has to go beyond the semantic correlation to help users find information and content naturally. The principle expands to the correlation of data between distinct points of interaction and apparatuses), due to taxonomy differences and lack of relation between representations of one same functionality. This was considered a major problem (gravity 3), as it is only possible to reach information about a saved place after 4 clicks.

Figure 10: wish list, instead of "saved", taking users to a list of saved places with no possibility of gathering further information.

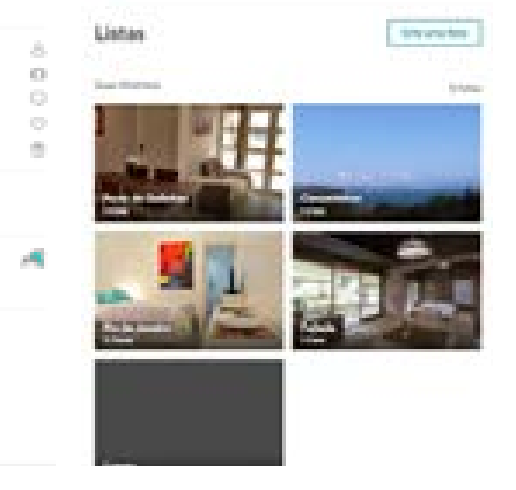

As a problem presented in all channels, while visiting apartment options, there is no indication of what is the neighborhood, unless inserted in the title of the place for renting (fig.11). This was considered to affect users' experience journey and related to heuristics 5 (correlation) and 3 (resilience: flexibility of the interaction flow and touch points in order to adequate to different users, different journey strategies and different contexts of use. The interactive ambiance and the system structure should be prepared to search, interaction and journey diverse strategies by different users, sometimes with distinct roles in the same journey).

Considering the importance of knowing detailed information about a place's location and its relation to mobility easiness, safety and distance to tourist points, reported from previous interviews with users (Renzi et al. 2018), the lack of information about location was marked as a major problem and could completely invalidate places for rent.

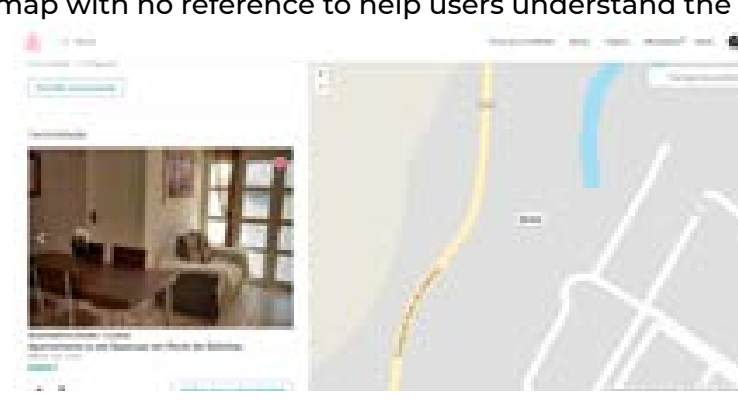

When using the Airbnb App for smartphone, evaluators also noted that when trying to reach details of any apartment's location information, it appears only as a pinpoint in a map, with no specifications. It becomes imperative for users to know the city of Rio de Janeiro, and its surroundings, geographically, in order to understand the area's proximity to transportation and tourist points. Evaluators related this to heuristics 3 (resilience), 6 (equivalency to cultural conventions) and 7 (visual intuitive content).

Also using the smartphone, results from search (showing options with titles and photos) do not follow any obvious pattern, which could cause confusion to users in understanding the relation between results and searched characteristics. Evaluators related this problem to heuristics 2 (consistency: the system has to present visual, typographic, information, actions and interaction consistency. If users utilize different devices to execute partial actions of the whole experience, each touchpoint access has to present the same rules and responses to actions, independent of the artifact that is being used) and 7 (visual intuitive content).

From selecting the best apartment option to start the reservation process using the desktop, it seems that the system is not prepared to complete the reservation easily. Evaluators point that it is necessary to explore the website to find how to do it. It was noted also to be difficult to understand the mechanism to send a message to the host.

\section{Conclusions}

Evidences during the evaluation show lack of strategy and lack of information architecture structure in the Airbnb system ecology to make it a pervasive crosschannel experience. Although an ecology system that can be reached from 3 different channels (desktop/laptop, smartphone and tablet), it seems that each channel was planned independently, without considering the possibility of users accessing their accounts and reservations from different devices while developing their experience journey.

From first steps of (1) dreaming moments, researching possibilities of vacation and lodge and analyzing apartment options for rent; and entering the (2) planning moments, gathering location and apartments information, to (3) booking and (4) experiencing moments, arriving at the rented apartment, users change devices all the time to interact with the system. The choice of a digital device to reach the system is influenced by external factors and experience contexts, as exposed by Sande et al.(2017) and Renzi (2017). And in a world of continuous advancements in pervasive experience, it is hard to think of users reaching the system through just one channel. 
The experts' analysis, using the set of 9 UX heuristics, show isolated interactions, making it difficult for users to change channels and continue the experience journey. The information is presented with inconsistency, hindering users to self-locate within the system and to finalize actions, inferring directly on heuristics of place-making, resilience, correlation and equivalency to cultural conventions.

The difficulties in finding relevant information, the breaking of users choices through the change of channel to interact with the system, resulted in complete loss of information, the necessity of redoing actions and going through longer paths to fulfil objectives, inferring directly to heuristics of place-making, reduction, correlation and visual intuitive content.

It is important to notice that although evaluators pointed problems of interaction related to isolated devices, the use of the 9 UX heuristics as basis for the evaluation enabled to pinpoint UX problems linked directly to cross-channel interaction experience. These cross-channel errors could not be traced using usability heuristics proposed for isolated devices, as they are problems surfaced through the change of interactive channels. This first experimentation with the UX heuristics is a step forward from previous research (Agner et al. 2017), regarding the analysis of the ecology system GloboPlay, where results showed that Nielsen and Molich's 10 heuristics (desktop interfaces) and Resmini and Rosatti's 5 heuristics (information architecture) were not enough to classify all problems noted by users in cooperative evaluation.

Parallel to this experimentation, the research group is testing the Airbnb system through traditional heuristic evaluation for future comparison. The tests are being executed separately, using Inostrozza et al. 12 heuristics for smartphone usability evaluation, Nielsen and Molich's 10 heuristics for desktop and tablet usability evaluation and think-aloud protocol. Each method is being executed with no contact among researchers, or exchange of information, to ensure no influence of data between all participants involved. Experts invited to participate in one of the evaluations do not participate in any other evaluation test. It is expected that all different evaluations will bring significant information to compare with these UX heuristic results and users' mental model map from interviews (Renzi et al. 2018).

It is important to include a few first impressions about the evaluation execution as part of the conclusion:

- In order to better analyze the cross-channel interaction, it is imperative to test all devices related to the system. But to establish a specific logical order for the experts to follow seems to be key for proper evaluation. Previous interview results (Renzi et al. 2018) evinced users do utilize different devices to build their user experience journey when planning their travels. The most common sequence of channel observed was used as basis to decide the order of devices and tasks to fulfill.

- Usually, when evaluating isolated devices using traditional heuristic evaluation experts are free to browse around to test every corner in search for problems. Continuous experience with heuristic evaluation and non-structured interviews with experts has shown that evaluators commonly start following a logic order of actions trying to simulate a real interaction sequence to fulfill objectives. For the crosschannel interaction evaluation, a sequence of tasks was presented to the evaluators. The tasks, simulating users' journey from dreaming moments to experience moments, were separated in steps to be executed partially by each device. Although the experts would browse around for problems, this idea was essential to help the experts focus on important users expectations and check the cross-channel interaction.
- At first, the idea of finishing the evaluation with a smartphone was to simulate the arrival of users to their destinations, through which most probably users would connect information about the rented apartment. As the testing proceeded, the idea of changing back to smartphone to complete the journey was important to help check the cross-channel interaction back TO the smartphone device, and not only FROM it.

- As the full evaluation needs to permeate different devices, the whole process is tiresome and takes longer than evaluating the system through just one isolated device. All participants expressed the need to pause in between tasks and get back refreshed to continue the evaluation. The longer time to fulfill the UX evaluation could be a problem to gather experts. The expressed need to pause by the evaluators can also imped the choice of face-to-face evaluation (Renzi and Freitas 2013) used for faster analysis.

- Since the cross-channel interaction permeates different devices, it is imperative that the invited experts have access to all types of devices involved in the evaluation. In this case, every invitee needed to have access to a desktop computer, to a smartphone and to a tablet. And obviously, each one of them had to install the Airbnb app for each device and register in order to perform the evaluation. If, in the near future, other types of devices are part of the system ecology to be evaluated, such as smart glasses, interactive surfaces, voice commanded ambiances (Renzi and Freitas 2015), all invited evaluators must have access to all apparatus in order to proper evaluate the crosschannel experience. Although it is preferred that each evaluator uses devices that they are familiar with and part of their daily interactions, if a restricted artifact is part of the evaluation, a proper place for testing should be provided.

- The UX heuristics evaluation can only be applied through the use of touchpoints of interaction, restricted to actual technological possibilities, as most of ecology systems have not yet fully integrated devices with interactive ambiences to create blended spaces. However, the set of heuristics is expected to be easily adapted to future interactive possibilities, integrating touch, voice, though and motion actions (Renzi and Freitas 2015).

As the experience of users permeate devices and part of their experience occurs outside the digital artifacts, even if still part of an experience ecology - such as stated by Sande et al. (2017) and Renzi (2017) and the blended spaces within the infosphere, exposed by Benyon and Resmini (2016) - to fully map possibilities within the whole UX journey and add the experience outside devices, other methods should be added to the investigation, such as interviews with users and the Flow-task observation method (Renzi 2013, 2017, 2018).

A further step, to be studied in the future, would be to broad the heuristic use to analyze the integration of cross-channel interactions with blended service design. 


\section{REFERENCES}

AGNER, L.; NECYK, B. J.; RENZI, A. B. Pervasive information architecture and media ecosystem: a Brazilian video on demand user experience. In: Design, User Experience, and Usability: Designing Pleasurable Experiences. $\mathrm{HCl}$ International 2017 Proceedings, part III. p. 570-580. Vancouver, BC, 2017.

APTED, T.; COLLINS, Anthony; KAY, Judy. Heuristics to Support Design of New Software for Interaction at Tabletops. Computer-Human Interactions, Boston, USA, 2009.

BENYON, D.; RESMINI, A. User Experience in Cross-channel Ecosystems. IN: 31st British Human Computer Interaction Conference, Sunderland, UK, 3-6 July, 2017.

HOW micro-moments are reshaping the travel customer journey. Link: <https://www. thinkwith google.com/marketing-resources/micro-moments/ micro-moments-travelcustomer-journey/>.

FLORIDI, L. Information: A Very Short Introduction. Oxford University Press, 2010.

FLORIDI, L. The Fourth Revolution - How the Infosphere is Reshaping Human Reality. Oxford

University Press, 2014

INOSTROZA, R.; RUSU, C.; RONCAGLIOLO, R.; RUSU, V. Usability heuristics for touchscreen based mobile devices. In: 9th International Conference on Information Technology - IEEE. Computer Society. Temuco, Chile, 2013.

INOSTROZA, R.; RUSU, C.; RONCAGLIOLO, R.; RUSU, V. Developing SMASH: A set of SMArtphone's uSability Heuristics. In: Computer Standards \& Interfaces 43, p.40-52. 2016

KOPETZ, H. Real-Time Systems. Real-Time Systems Series. Springer, 2011

NETO, E. V.; CAMPOS, F. F.C. Evaluating the usability on multimodal interfaces: a case study on tablets applications. Design, User Experience, and Usability. Theories, Meth-ods, and Tools for Designing the User Experience. DUXU, part 1,pp.484-495. Crete, Greece, 2014.

NIELSEN, J. Characteristics of Usability Problems Found by Heuristic Evaluation https://www.nngroup.com/articles/usability-problems-found-by-heuristic-evaluation/. 1995.

NIELSEN, J. 10 usability heuristics for interface design. < www.nngroup.com/ articles / ten-usability-heuristics>. 1995

NIELSEN, J. How to Conduct a Heuristic Evaluation. https://www.nngroup.com/articles/ how-to-conduct-aheuristic-evaluation/ 1995
RENZI, A. B.; FREITAS, Sydney. Aplicação de avaliação heurística para diagnosticas problemas de usabilidade na procura e compra de livros em livrarias online. Revista Ergodesign \& HCl. N1. vol.1. year 1. Rio de Janeiro, Brazil. p. 10-21. 2013

RENZI, A. B. Experiência do ususário: a jornada de Designers nos processos de gestão de suas empresas de pequeno porte utilizando sistema fantasiado em ecossistema de interação cross-channel. Thesis. 239p. Escola Superior de Desenho Industrial. Rio de Janeiro, Brazil, 2016

RENZI, A. B; FREITAS, Sydney. Delphi method to explore future scenario possibilities on technology and HCl. Human-Computer Interactions International. DUXU. pp.644653. Springer. Los Angeles, CA, 2015

RENZI, A. B. 2017. Experiência do usuário: construção da jornada pervasiva em um ecossistema. SPGD2017. Rio de Janeiro, Brazil, 2017.

SANDE, Axel; RENZI, A. B.; SCHNAIDER, Silvia. 2017. Experience, usability and the sense of things. In:Design, User Experience, and Usability: Designing Pleasurable Experiences. HCl International 2017 Proceedings, part II. p.77-86. Vancouver, BC. 2017.

RENZI, A. B. The use of Task-flow observation to map users' experience and interaction touchpoints. In: Applied Human Factors and Ergonomics 2018. Orlando, FL, USA. 2018.

RENZI, A. B.; MUNIZ, João Vitor Acácio; FIÚZA, Juliana. Experiência do usuário pervasiva no planejamento de viagens: mapeando modelo mental e criando personas. SPGD2018. Rio de Janeiro, RJ, 2018

RENZI, A. B. UX Heuristics for cross-channel interactive scenarios. In: Design, User Experience, and Usability: Theory, Methodology, and Management, pp.481- $491 . \mathrm{HCl}$ International 2017 Proceedings, Vancouver, BC, 2017

RESMINI, A.; ROSATTI, L. Pervasive information architecture-Designing cross-channel user experiences. Morgan Kaufmann - inprint of Elsevier. Burlington, MA. 2011

RESMINI, A.; LACERDA, F. The Architecture of Cross-channel Ecosystems - From convergence to experience. Proceedings of the 8th International ACM Conference on the Management of Digital Ecosystems (MEDES'16). 2016.

\section{Acknowledgements}

We would like to thank all specialists who volunteered for this UX evaluation first experiment. The evaluation clarified many questions and surfaced new important ones. 